\section{The homeobox protein Prox1 is a negative modulator of ERR $\alpha /$ PGC-1 $\alpha$ bioenergetic functions}

\author{
Alexis Charest-Marcotte, ${ }^{1,2}$ Catherine R. Dufour, ${ }^{1}$ \\ Brian J. Wilson, ${ }^{1}$ Annie M. Tremblay, ${ }^{1,2}$ \\ Lillian J. Eichner, ${ }^{1,2}$ Daniel H. Arlow, ${ }^{3,4,5}$ \\ Vamsi K. Mootha, ${ }^{3,4,5}$ \\ and Vincent Giguère ${ }^{1,2,6,7,8}$ \\ ${ }^{1}$ Goodman Cancer Centre, McGill University, Montréal, \\ Québec H3A 1A3, Canada; ${ }^{2}$ Department of Biochemistry, \\ McGill University, Montréal, Québec H3G 1Y6, Canada; \\ ${ }^{3}$ Department of Systems Biology, Massachusetts General \\ Hospital, Cambridge, Massachusetts 02142, USA; ${ }^{4}$ Centre for \\ Human Genetic Research, Massachusetts General Hospital, \\ Cambridge, Massachusetts 02142, USA; ${ }^{5}$ Broad Institute of \\ Massachusetts Institute of Technology/Harvard, Cambridge, \\ Massachusetts 02142, USA; ${ }^{6}$ Department of Medicine, McGill \\ University, Montréal, Québec H3G 1Y6, Canada; ${ }^{7}$ Department \\ of Oncology, McGill University, Montréal, Québec H3G 1Y6, \\ Canada
}

Estrogen-related receptor $\alpha(E R R \alpha)$ and proliferatoractivated receptor $\gamma$ coactivator-1 $\alpha(\mathrm{PGC}-1 \alpha)$ play central roles in the transcriptional control of energy homeostasis, but little is known about factors regulating their activity. Here we identified the homeobox protein prospero-related homeobox 1 (Prox1) as one such factor. Prox1 interacts with ERR $\alpha$ and PGC-1 $\alpha$, occupies promoters of metabolic genes on a genome-wide scale, and inhibits the activity of the ERR $\alpha /$ PGC-1 $\alpha$ complex. DNA motif analysis suggests that Prox1 interacts with the genome through tethering to $\mathrm{ERR} \alpha$ and other factors. Importantly, ablation of Prox1 and ERR $\alpha$ have opposite effects on the respiratory capacity of liver cells, revealing an unexpected role for Prox1 in the control of energy homeostasis.

Supplemental material is available at http://www.genesdev.org. Received October 5, 2009; revised version accepted January 21, 2010.

Regulation of energy homeostasis involves elaborate biochemical pathways that have evolved to react to the metabolic needs of the organism in response to specific physiological states. While homeostatic regulation is generally under hormonal control and achieved through allosteric control and post-translational modifications of metabolic enzymes for immediate needs, organ-specific requirements and lasting adaptation require regulation of

[Keywords: ChIP-on-chip; homeobox; mitochondrial respiration; nuclear receptor; regulon]

${ }^{8}$ Corresponding author.

E-MAIL vincent.giguere@mcgill.ca; FAX (514) 843-1478.

Article published online ahead of print. Article and publication date are online at http://www.genesdev.org/cgi/doi/10.1101/gad.1871610. metabolic genes at the transcriptional level via the action of diverse classes of transcription factors and coregulatory proteins (Desvergne et al. 2006; Feige and Auwerx 2007). Among those factors, the orphan nuclear receptor estrogenrelated receptor $\alpha(\mathrm{ERR} \alpha, \mathrm{NR} 3 \mathrm{~B} 1)$ and the coregulator peroxisome proliferator-activated receptor $\gamma$ coactivator$1 \alpha$ (PGC-1 $1 \alpha)$ have been shown to play a predominant role in controlling several aspects of energy metabolism, most notably mitochondrial biogenesis and oxidative phosphorylation (Oxphos) (Lin et al. 2005; Giguère 2008).

Prospero-related homeobox 1 (Prox 1 ) is a transcription factor essential for the development of numerous tissues, including the liver (Sosa-Pineda et al. 2000; Burke and Oliver 2002; Dudas et al. 2006). In particular, Prox1 plays a critical role in determining the fate of lymphatic endothelial cells and, consequently, Prox1-null embryos are devoid of lymphatic vasculature and die in utero at approximately embryonic day 14.5 (Wigle and Oliver 1999; Johnson et al. 2008). Prox1 haploinsufficient mice also display lymphatic vascular defects that have been proposed to lead to adult-onset obesity via the promotion of adipogenesis and increased fat storage in lymphaticrich regions (Harvey et al. 2005). Proxl is also known to regulate the activity of a specific subset of nuclear receptors (Qin et al. 2004; Song et al. 2006; Lee et al. 2009; Yamazaki et al. 2009). Of particular interest, Proxl was shown to regulate the activity of hepatocyte nuclear factor $4 \alpha$ (HNF4 $\alpha$, NR2A1) and liver receptor homolog-1 (LRH-1, NR5A2) on the CYP7A1 and PCK1 promoters, suggesting a possible role for Proxl in the regulation of bile acid synthesis and gluconeogenesis in the liver (Qin et al. 2004; Song et al. 2006). Whether Prox1 plays a more comprehensive role in the regulation of energy metabolism is currently unknown.

\section{Results and Discussion}

Prox1 interacts with and modulates the activity of the $E R R \alpha / P G C-1 \alpha$ complex

An automated yeast two-hybrid interaction screen previously identified fragments of Proxl as interactors of $E R R \alpha$ (Albers et al. 2005). We first sought to validate the physiological significance of this interaction by performing coimmunoprecipitation experiments with endogenous proteins present in the mouse liver. As observed in Figure 1A, ERR $\alpha$ could be detected in extract immunoprecipitated with a Prox 1 antibody, while Prox 1 could be detected in liver lysate immunoprecipitated with an $\operatorname{ERR} \alpha$ antibody. As expected, but not shown previously, a potent in vivo interaction was observed in the mouse liver between ERR $\alpha$ and PGC-1 $\alpha$ (Fig. 1A). Prox1 can be also found in a complex with PGC- $1 \alpha$. Direct interactions were detected between Prox 1 and both ERR $\alpha$ and PGC- $1 \alpha$ via in vitro GST pull-down experiments (Fig. 1B). Only the $\mathrm{N}$ terminus of Prox 1 binds to PGC- $1 \alpha$, while both the $\mathrm{N}$ terminus and $\mathrm{C}$ terminus of Proxl interact with ERR $\alpha$. Proxl interacts with ERR $\alpha$ solely through its DNAbinding domain (DBD) (Fig. 1C). Indeed, an altered Prox1 protein containing inactivation mutations for the two putative LxxLL interaction motifs (NR1/2mut) known to be required for the interaction with LRH1 and HNF4 $\alpha$ (Qin et al. 2004; Song et al. 2006) was able to interact physically with ERR $\alpha$. Finally, Prox1 was found to interact 
A

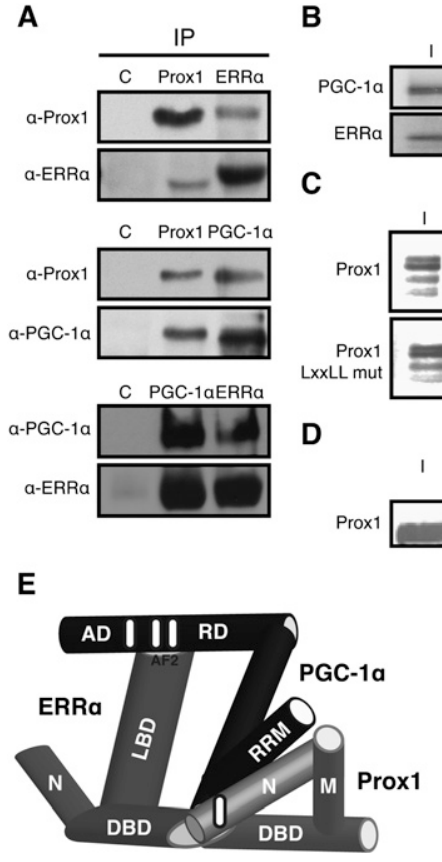

G

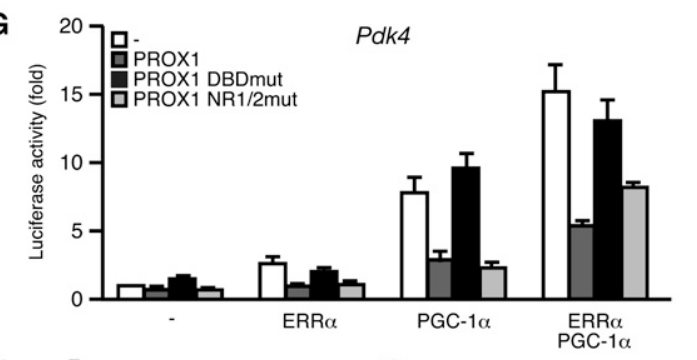

H

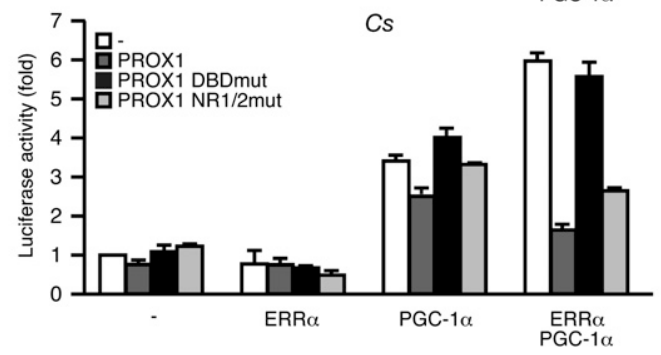

Figure 1. Proxl interacts with and influences the transcriptional activity of ERR $\alpha$ and PGC- $1 \alpha$. (A) Proxl, ERR $\alpha$, and PGC- $1 \alpha$ interact in vivo. Lysates from mouse liver were subjected to immunoprecipitation and immunoblot analyses with the indicated antibodies. (B) Direct interactions between Proxl, PGC- $1 \alpha$, and ERR $\alpha$. In vitro translated ERR $\alpha$ and PGC-1 $\alpha$ were subjected to pull-down analysis with GST-Prox 1 fragments. (N) N terminus; (M) middle; (C) C terminus. (C) Prox1 interacts with the DNA-binding domain (DBD) of ERR $\alpha$. In vitro translated Prox 1 and a LxxLL 1/2 mutant were subjected to pull-down analysis with GST-ERR $\alpha$ fragments. (FL) Full-length; (N) N terminus; (LBD) ligand-binding domain. $(D)$ Proxl interacts with a new functional domain of PGC- $1 \alpha$. In vitro translated Prox1 with GST-PGC-1 $\alpha$ fragments. (E) Schematic representation of a potential trimeric interaction between Proxl, ERR $\alpha$, and PGC-1 $\alpha$. (AD) Activation domain; (RD) repression domain; (RRM) RNA recognition motif; (AF-2) activation function 2; (white bars) LxxLL motifs. (F) Re-ChIP experiments performed in the mouse liver on the Pdk4 promoter using either anti-ERR $\alpha$ or antiProxl antibodies in a serial manner. $(G)$ Effects of wild-type and mutant Proxl proteins on the transcriptional activity of ERR $\alpha$ and PGC- $1 \alpha$. The Pdk4 promoter-luciferase reporter gene was cotransfected in HepG2 cells with empty vector (-), ERR $\alpha$, PGC- $1 \alpha$, or a combination of both expression vectors in the presence or absence of wild-type or mutant Proxl. $(H)$ Same assay as in $G$ using the $C$ s promoter as the reporter gene. with PGC- $1 \alpha$ via a domain comprised of residues 483-631, a domain without a previously assigned function (Fig. 1D). A schematic representation of the potential ERR $\alpha / \operatorname{Prox} 1 /$ PGC- $1 \alpha$ trimeric complex is shown in Figure 1E.

We next tested whether ERR $\alpha$ and Proxl could form a complex on chromatin by performing a serial chromatin immunoprecipitation (ChIP) experiment in the liver at the Pdk4 promoter. As shown in Figure $1 \mathrm{~F}$, re-ChIP for ERR $\alpha$ generated further enrichment following an initial ChIP for Proxl, while the converse re-ChIP experiment generated even more enrichment for Proxl at the Pdk4 promoter. Next, the Pdk4 promoter was fused to the luciferase reporter gene, and the construct was cotransfected in HepG2 cells together with expression vectors for ERR $\alpha$ and PGC-1 $1 \alpha$. As shown in Figure 1G, introduction of Prox1 decreased both the basal and ERR $\alpha$-induced and/or PGC$1 \alpha$-induced luciferase activity. In agreement with the physical interaction data, the altered Proxl protein containing inactivation mutations of the two putative LxxLL interaction motifs (NR1/2mut) still retains a repressive effect, while a Prox1 DBD mutant is no longer functional for $\mathrm{ERR} \alpha$ target gene repression. Similar results were obtained when using reporter constructs linked to the Cs, Cycs, and ApoC3/Apoa4 promoters (Fig. 1H; Supplemental Fig. S1).

\section{ChIP-on-chip analyses identify a genomic relationship between ERR $\alpha$ and Prox1}

We next performed genome-wide location analyses to assess the extent of the functional interaction between Proxl and ERR $\alpha$. To be able to directly relate the binding events to a specific target gene, we performed two distinct ChIP-on-chip experiments using tiled genomic DNA arrays covering the extended promoter regions $(-5.5$ to $+2.5 \mathrm{~kb}$ from transcriptional start sites $)$ of $\sim 17,000$ mouse genes and antibodies specific to ERR $\alpha$ and Prox1. Analysis of the ChIP-on-chip data sets identified 2479 and 2266 high-confidence binding sites mapping to the promoters of 2373 and 2069 genes in the mouse liver for ERR $\alpha$ and Prox1, respectively (Fig. 2A; Supplemental Tables S1, S2). Comparison of the data sets from both factors revealed that a total of $937 \mathrm{ERR} \alpha$ target genes are also targets of Proxl (39.5\% of all ERR $\alpha$ targets) (Fig. 2A). Standard ChIP validation and examination of the bound segments revealed four different classes of promoter regions targeted by the two factors (Fig. 2B,C). Of the 937 promoters shared by ERR $\alpha$ and Prox1, 527 contain a common segment bound by both factors ( $22 \%$ of all ERR $\alpha$ targets) (Fig. 2B). Considering that PGC- $1 \alpha$ can interact with both Proxl and ERR $\alpha$ (Fig. 1), we next tested whether PGC- $1 \alpha$ associates with chromatin at sites bound specifically by Prox 1 or $\mathrm{ERR} \alpha$, or only when both factors are present. Standard ChIP analysis revealed that PGC-1 $\alpha$ is recruited at DNA segments recognized by Prox 1 or ERR $\alpha$ (Fig. 2B), consistent with the observation that PGC- $1 \alpha$ interacts directly with both partners (Fig. 1B).

The results of the ChIP-on-chip experiments were then analyzed using motif-finding algorithms. First, we searched for known transcription factor-binding motifs that were enriched in bound segments, and found that, in both ERR $\alpha$-specific and ERR $\alpha /$ Proxl shared segments, the most enriched motifs were ERREs (Supplemental Table S3). In agreement with our previous analysis (Dufour et al. 2007), CREB-binding motifs were also enriched in ERR $\alpha$-specific segments, suggesting that the functional interaction between $\mathrm{ERR} \alpha$ and CREB observed 
A

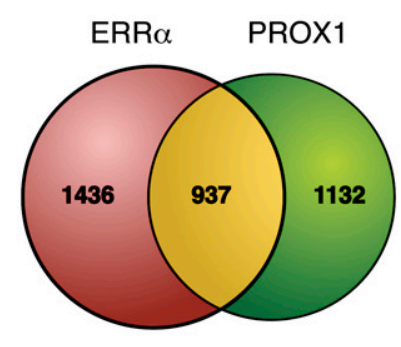

C

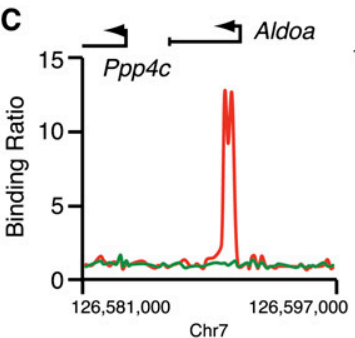

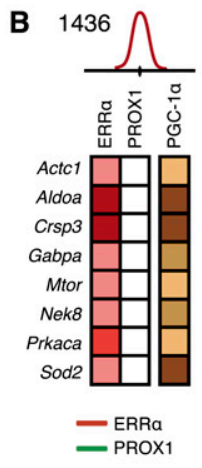
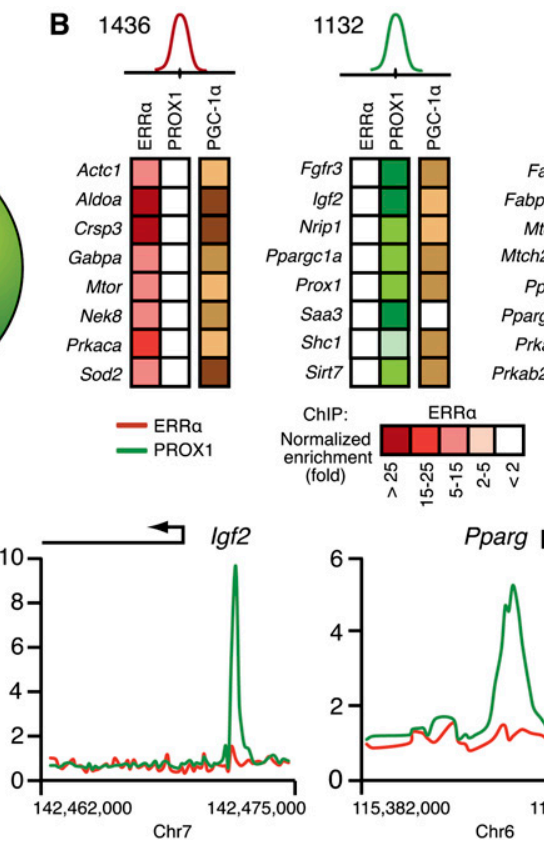

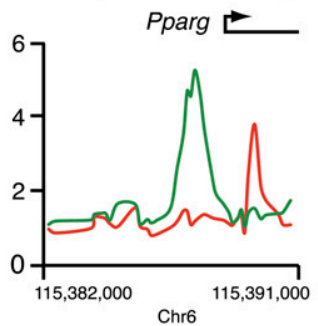

Figure 2. Genome-wide promoter occupancy of ERR $\alpha$ and Proxl in mouse liver. (A) Venn diagrams illustrating the overlap in ERR $\alpha($ red) and Proxl (green) direct target genes from ChIP-on-chip analyses in the mouse liver. (B) Standard ChIP validation of a subset of ERR $\alpha$-enriched (red) and Prox1-enriched (green) segments. Occupancy of PGC-1 $\alpha$ on these selected DNA segments bound by ERR $\alpha$, Prox 1, or both factors as assayed by standard ChIP is also shown. $(C)$ Representative binding profiles of ERR $\alpha$ (red line) and Proxl (green line) on specific or common target extended promoters containing either distinct or overlapping binding sites.

in the heart is also operational in the liver. On the other hand, analysis of Proxl-specific segments revealed enrichment of HNF4 $\alpha$ - and C/EBP $\beta$-binding sites (Supplemental Table S3). Next, we tested a dictionary of 741 motifs identified by conservation across four mammals (Xie et al. 2005) for enrichment in bound segments using motifADE (Mootha et al. 2004), and again found that the most significantly enriched motifs in both ERR $\alpha$-specific and ERR $\alpha /$ Prox 1 shared segments were ERREs (Table 1). However, while several motifs were enriched in Proxlspecific segments, we were not able to identify with high confidence any homeobox-like motifs.

\section{The ERR $\alpha$ regulon}

We next evaluated the biological processes associated with genes with promoter regions that are enriched specifically for ERR $\alpha$, Prox 1 or both factors. As expected from previous work (Mootha et al. 2004; Schreiber et al. 2004; Dufour et al. 2007; Sonoda et al. 2007; Deblois et al. 2009), analysis showed ERR $\alpha$ target genes highly enriched for processes linked to metabolism (Fig. 3A; Supplemental Fig. S2). ERR $\alpha /$ Prox1 shared genes were also significantly enriched for the tricarboxylic acid (TCA) cycle as well as pyruvate metabolism. Similarly, Prox1specific genes were significantly enriched for glycolysis/ gluconeogenesis and pyruvate metabolism, and also for bile acid, histidine, and purine metabolism, but were virtually absent for Oxphos and the TCA cycle (Supplemental Fig. S3). Remarkably, precise assignment of ERR $\alpha$ and Proxl target genes to pathways involved in energy production revealed that $\operatorname{ERR} \alpha$ binds to the extended promoter regions of genes encoding enzymes at every step in the glycolytic pathway, pyruvate metabolism, and TCA cycle (Fig. 3B). This cluster of functionally linked genes is subsequently referred to as the ERR $\alpha$ bioenergetic regulon. ERR $\alpha$-bound segments can also be found in the promoter regions of a large number of genes encoding proteins that constitute the five complexes of the Oxphos pathway (Fig. 3B). The ERR $\alpha$ bioenergetic regulon also includes a significant number of genes whose extended promoter regions are bound by Prox 1, most notably genes encoding enzymes at key entry points in energy production pathways such as G6pc, Ldhb, Pdk4, Pcx, Pck1, Cs, and Fh1.

\section{Divergent regulation of bioenergetic functions by ERR $\alpha$ and Prox 1}

We then examined the role of ERR $\alpha$ and Proxl in the regulation of bioenergetic functions in HepG2 cells. We first demonstrated that $\mathrm{ERR} \alpha$, Proxl, and PGC- $1 \alpha$ are indeed present and can interact with each other in HepG2 cells (Supplemental Fig. S4). We also showed, using siRNAs to silence ERR $\alpha$ and Proxl expression, that both factors can regulate a subset of metabolic genes indentified as ERR $\alpha$ target genes in the mouse liver (Supplemental Fig. S5). The HepG2 expression data set indicates that ERR $\alpha$ and Proxl have, in general, contrasting effects on the expression of genes involved in bioenergetic pathways. We next measured in vivo cellular respiration and glycolytic rates in HepG2 cells in the presence or absence of two specific sets of siRNAs against either ERR $\alpha$ or Prox1 (Fig. 4A; Supplemental Fig. S6). HepG2 cells treated with control siRNA displayed an expected cellular respiration rate (OCR) profile that was first inhibited by addition of the ATP synthase (Complex V) inhibitor oligomycin, then enhanced with the uncoupling agent p-trifluoromethoxy carbonyl cyanide phenyl hydrazone (FCCP), and repressed again with the Complex I 
Table 1. De novo enriched motifs in ERR $\alpha, E R R \alpha /$ Prox1, or Prox1 target promoters at $\mathrm{P}<0.01$

\begin{tabular}{|c|c|c|c|c|}
\hline Motif & Annotation & $\operatorname{ERR} \alpha$ & ERR $\alpha / \operatorname{Prox} 1$ & Proxl \\
\hline TGACCTY & NR & $5.75 \times 10^{-144}$ & $1.58 \times 10^{-27}$ & NS \\
\hline TGACCTTG & SF1 & $7.10 \times 10^{-132}$ & $1.06 \times 10^{-13}$ & NS \\
\hline TGACCT & NR & $1.64 \times 10^{-113}$ & $3.75 \times 10^{-24}$ & NS \\
\hline GTGACCY & NR & $5.08 \times 10^{-47}$ & $1.08 \times 10^{-3}$ & NS \\
\hline GTGWMCTT & SF1 & $8.04 \times 10^{-40}$ & NS & NS \\
\hline GTGNCMTTG & SF1 & $3.98 \times 10^{-38}$ & $1.50 \times 10^{-4}$ & NS \\
\hline YSACCWTGG & SF1 & $3.71 \times 10^{-30}$ & $9.00 \times 10^{-3}$ & NS \\
\hline CTGWCCTTNR & NR & $2.02 \times 10^{-25}$ & NS & NS \\
\hline GAAGGTMR & NR & $4.96 \times 10^{-25}$ & $1.82 \times 10^{-3}$ & NS \\
\hline GGTNACNTTG & CREB & $1.09 \times 10^{-21}$ & NS & NS \\
\hline GAAKKTCA & - & $3.48 \times 10^{-17}$ & NS & NS \\
\hline RGGTGACNY & CREB & $8.78 \times 10^{-16}$ & NS & NS \\
\hline GGTGACNT & CREB & $4.34 \times 10^{-15}$ & NS & NS \\
\hline YTTGAMCTT & NR & $8.81 \times 10^{-14}$ & NS & NS \\
\hline GTGRNYTTGG & SF1 & $2.25 \times 10^{-12}$ & NS & NS \\
\hline YYTTGACCY & NR & $1.80 \times 10^{-11}$ & $2.91 \times 10^{-4}$ & NS \\
\hline YGTCCTTGT & - & $4.19 \times 10^{-5}$ & NS & NS \\
\hline TGAMCTTT & NR & $5.98 \times 10^{-4}$ & $5.64 \times 10^{-4}$ & $8.09 \times 10^{-6}$ \\
\hline AGGTGA & MYOD & $1.89 \times 10^{-3}$ & NS & NS \\
\hline RAGTGACNY & CREB & $2.47 \times 10^{-3}$ & NS & NS \\
\hline TGCCAAR & NF1 & NS & $5.52 \times 10^{-4}$ & $3.46 \times 10^{-10}$ \\
\hline RATCRATA & CDP & NS & NS & $3.31 \times 10^{-6}$ \\
\hline GGACTTY & NFKB, TEF1 & NS & $6.02 \times 10^{-3}$ & $5.55 \times 10^{-5}$ \\
\hline TAANMAAG & NKX61, LHX3 & NS & NS & $5.93 \times 10^{-4}$ \\
\hline AAAYATT & FOXJ2, TBP & NS & NS & $7.63 \times 10^{-4}$ \\
\hline TTCYNRGAA & IK1, STAT & NS & NS & $2.03 \times 10^{-3}$ \\
\hline TTGRN $_{6}$ TCCAR & - & NS & NS & $4.88 \times 10^{-3}$ \\
\hline GCCARGAA & ETS2, ELK1 & NS & NS & $5.09 \times 10^{-3}$ \\
\hline TTTNAAC & POU3F2, OCT & NS & NS & $5.91 \times 10^{-3}$ \\
\hline
\end{tabular}

Nuclear receptor (NR) includes ERR $\alpha$ (NR3B1), estrogen receptor $\alpha$ (NR3A1), GNCF (NR6A1), thyroid hormone receptor $\alpha$ (NR1A1), RORA (NR1F1), and COUP-TF (NR2F1). (NS) Not significant.

inhibitor rotenone (Fig. 4B; Supplemental Fig. S6). HepG2 cells lacking ERR $\alpha$ and Proxl were found to have a decreased and increased response to FCCP relative to control cells, respectively. The FCCP-stimulated OCRs show that HepG2 cells lacking ERR $\alpha$ display impaired mitochondrial function, and that cells lacking Prox1 have a greater cellular respiratory capacity. Our experiment also revealed that cells treated with $\operatorname{ERR} \alpha$ siRNA were found to have significantly lower extracellular acidification rates following oligomycin and FCCP addition (Fig. 4C; Supplemental Fig. S6), indicating that the presence of $\mathrm{ERR} \alpha$ is indispensable for the ability of HepG2 cells to switch from oxidative to glycolytic metabolism.

The work presented herein not only extends the repertoire of nuclear receptors with which Proxl physically interacts to include $\operatorname{ERR} \alpha$, but broadens these functional interactions to a coactivator protein, PGC- $1 \alpha$. Furthermore, the identification of ERR $\alpha$ and Prox 1 target genes in the mouse liver establishes a unique relationship between the two factors at both a genomic and functional level. Our study also demonstrates the efficacy of the ChIP-on-chip on promoter array approach to define the regulon of a eukaryotic transcription factor. Indeed, we show that ERR $\alpha$ binds to the extended promoter regions of genes encoding virtually all enzymes involved in glycolysis, pyruvate metabolism, and the TCA cycle. The relevance of the metabolic role of ERR $\alpha$ and Prox 1 was further probed by monitoring in vivo cellular respiration and glycolytic rates in HepG2 liver cells upon
ERR $\alpha$ and Proxl knockdown. The results indicate that ERR $\alpha$ and Proxl have opposite effects on the respiratory capacity of liver cells, and that the presence of ERR $\alpha$ is essential for the switch to glycolysis when mitochondrial Oxphos is unable to meet the energy demands of the cell.

One of the unexpected elements of this study is the identification of the ERR $\alpha$ bioenergetic regulon. In prokaryotes and lower eukaryotes, regulons represent a widespread mechanism to coordinate the concurrent expression of a group of genes by a common transcription factor. In higher eukaryotes, the complexity of gene regulation is often linked to a multitude of extracellular signals, and this may preclude the use of a common factor to regulate all genetic components of an integrated biochemical pathway. Remarkably, ERR $\alpha$ occupies the extended promoter regions of practically all genes encoding enzymes of three well-defined biochemical pathways involved in the generation of energy from glucose. The potential to regulate linked biochemical pathways involved in energy metabolism likely evolved to ensure a coordinated increase in energy output in response to physiological stressors that are known to up-regulate the expression of the ERR $\alpha$ protein ligands PGC- $1 \alpha$ and/or PGC- $1 \beta$.

Proxl is a homeobox protein, and, as expected, most studies probing its functions focused on embryonic development (Wigle and Oliver 1999; Sosa-Pineda et al. 2000; Kamiya et al. 2008). The results of our study demonstrate a novel and comprehensive role for Prox1 
A

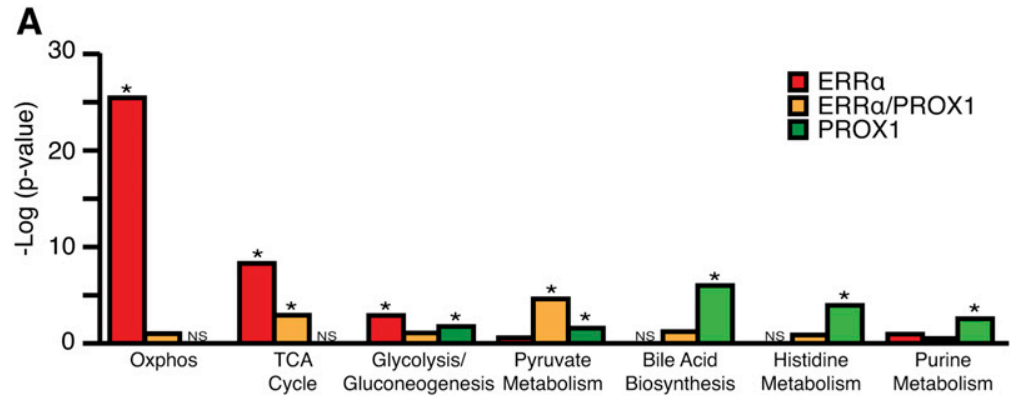

B

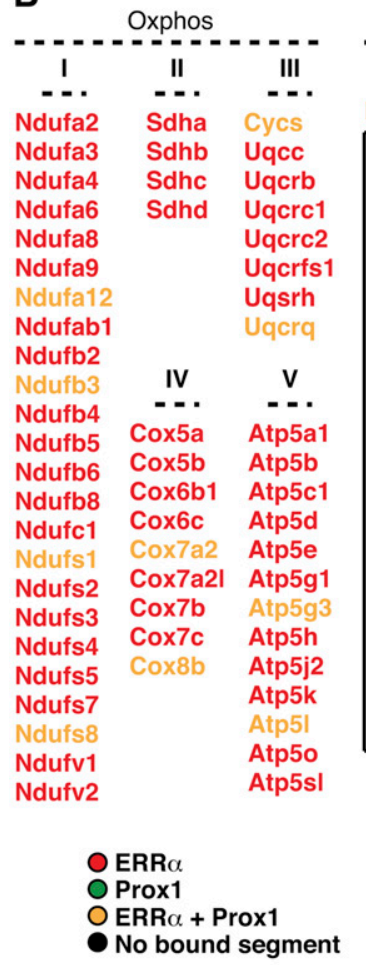

Glycolysis/

Gluconeogenesis

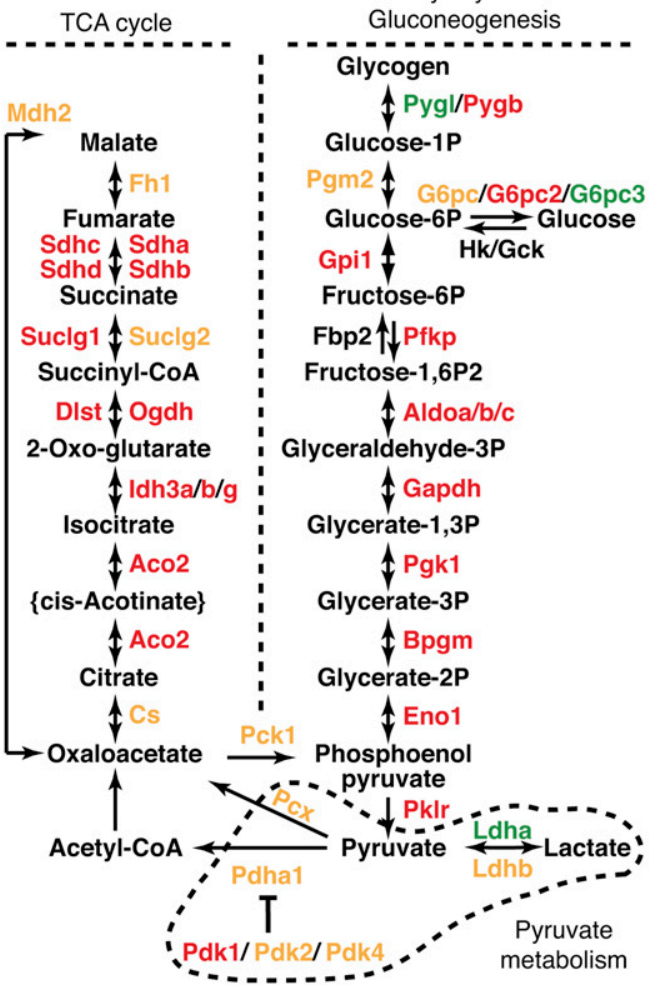

In conclusion, we demonstrated that Prox 1 acts as a negative modulator of the $\mathrm{ERR} \alpha /$ PGC$1 \alpha$ axis, and, as such, regulates a broad transcriptional program implicated in the control of energy homeostasis in the liver that includes a newly defined bioenergetic regulon controlled by ERR $\alpha$. Our results also reinforce the concept that the ERR isoforms are essential factors controlling the transition from carbohydrate-based to lipid-based oxidative metabolism (Alaynick et al. 2007; Dufour et al. 2007). The physiological significance of these findings is further highlighted by the recent identification of Proxl as a genetic locus implicated in fasting glucose homeostasis and increased risk for type II diabetes (Dupuis et al. 2010). Thus, the identification of Proxl as an important regulator of the ERR $\alpha / \mathrm{PGC}-1 \alpha$ axis suggests that novel strategies for managing diseases involving long-term energy imbalance can be envisaged.

\section{Materials and methods}

\section{Animals}

Adult male C57BL/6J mice were housed and fed standard chow in the animal facility at the McGill University Health Center. In all experiments involving mouse livers, mice were sacrificed during the day at ZT (Zeitgeber time) 4 .

\section{Cell culture and reporter assays}

Luciferase constructs and reporter assays in HepG2 cells are described in the Supplemental Material.

Coimmunoprecipitation, immunoblotting, and GST pull-down assays

Coimmunoprecipitation, Western blot, and GST pull-down assays involving ERR $\alpha$, PGC- $1 \alpha$, and Prox 1 are described in the Supplemental Material.

Figure 3. The ERR $\alpha$ bioenergetic regulon. (A) Enrichment of canonical metabolic pathways in the ChIP-on-chip target genes determined to be common (yellow) or specific to either ERR $\alpha$ (red) or Proxl (green). (NS) Not significant; $\left(^{\star}\right) P<0.05$. $(B)$ ChIP-on-chip direct target genes specific to ERR $\alpha$ (red) or Proxl (green) or shared by both factors (yellow) involved in metabolic pathways are shown. All genes involved in glycolysis, pyruvate metabolism, and the TCA cycle are targets of ERR $\alpha$, a cluster of genes defining the ERR $\alpha$ bioenergetic regulon. Genes labeled in black were not identified as being enriched by either ERR $\alpha$ or Prox1.

in the direct control of energy homeostasis. Prox1 thus joins HNF1 $\beta$ as a rare example of a homeobox-containing factor involved directly in the transcriptional regulation of metabolism (Desvergne et al. 2006). However, our computational analysis of sequence-specific DNA recognition suggests that Proxl's sequence-specific binding to the genome in the adult liver is likely through interaction with other factors, most prominently ERR $\alpha, C / E B P \beta$, and HNF4 $\alpha$. Our results thus suggest that Proxl's main mode of action in this context is that of a corepressor. While the exact molecular mechanism by which Prox 1 exerts its repressor effect remains to be determined, preliminary analysis indicates that immunoprecipitated Prox 1 is not associated with histone deacetylase (HDAC) activity (Supplemental Fig. S7).
ChIP, re-ChIP, ChIP-on-chip, ChIP-qPCR, and functional analysis of target genes

Mouse liver ERR $\alpha$, Proxl, and PGC- $1 \alpha$ ChIP, serial ChIP, and genome-wide location analyses performed using Agilent extended promoter arrays are described in the Supplemental Material. Bed files are available on request. Primer sequences used for ChIP-qPCR are shown in Supplemental Table S4. Ingenuity Pathway Analysis software (Ingenuity Systems, http://www.ingenuity.com) was used for functional analysis of target genes (see the Supplemental Material).

\section{Computational motif discovery}

Enriched motifs within ERR $\alpha$ and Proxl ChIP-on-chip targets were identified using MOTIFCLASS (Smith et al. 2006) and motifADE (Mootha et al. 2004) as described in the Supplemental Material.

\section{siRNA, $q R T-P C R$, and extracellular flux (XF) analysis}

ERR $\alpha$ and Prox 1 knockdown experiments in HepG2 cells with subsequent qRT-PCR and bioenergetic analysis using a Seahorse Extracellular Flux (XF24) Analyzer are described in the Supplemental Material. Primers used for qRT-PCR are shown in Supplemental Table S5. 
A

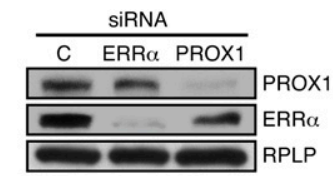

B

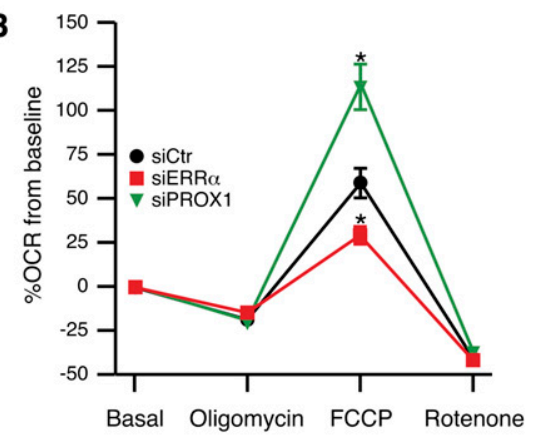

C

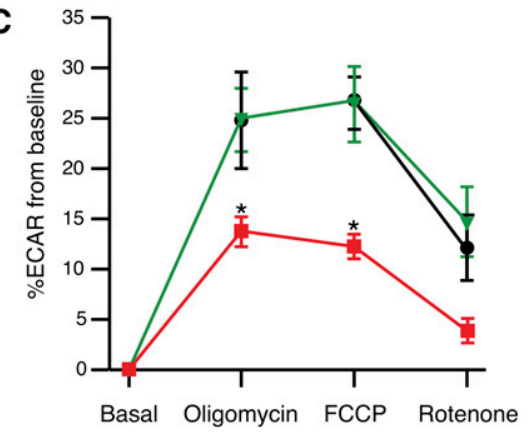

Figure 4. Divergent regulation of mitochondrial functions by ERR $\alpha$ and Prox1. (A) Western blot analysis on lysates prepared from the HepG2 knockdown samples is shown with the respective antibodies as indicated. Detection of RPLP was used a control. $(B, C)$ Cellular oxygen consumption $(B)$ and extracellular acidification rates $(C)$ were measured in intact HepG2 cells treated with either control siRNA or an siRNA against ERR $\alpha$ or Proxl. Rates determined following sequential addition of oligomycin, FCCP, and rotenone were taken from an average of two measurements and are expressed as a percentage of the baseline rates. $\left(^{\star}\right) P<0.05$.

\section{Acknowledgments}

We thank Carlo Ouellet for skillful technical assistance and Dr. Julie St-Pierre for careful reading of the manuscript. This work was supported by grants from the Canadian Institutes for Health Research (MOP-84227 and MOP-77763) and the American Diabetes Association/Smith Family Foundation.

\section{References}

Alaynick WA, Kondo RP, Xie W, He W, Dufour CR, Downes M, Jonker JW, Giles W, Naviaux RK, Giguère V, et al. 2007. ERR $\gamma$ directs and maintains the transition to oxidative metabolism in the post-natal heart. Cell Metab 6: 16-24.

Albers M, Kranz H, Kober I, Kaiser C, Klink M, Suckow J, Kern R, Koegl M. 2005. Automated yeast two-hybrid screening for nuclear receptorinteracting proteins. Mol Cell Proteomics 4: 205-213.

Burke Z, Oliver G. 2002. Proxl is an early specific marker for the developing liver and pancreas in the mammalian foregut endoderm. Mech Dev 118: 147-155.

Deblois G, Hall JA, Perry MC, Laganiere J, Ghahremani M, Park M, Hallett M, Giguere V. 2009. Genome-wide identification of direct target genes implicates estrogen-related receptor $\alpha$ as a determinant of breast cancer heterogeneity. Cancer Res 69: 6149-6157.

Desvergne B, Michalik L, Wahli W. 2006. Transcriptional regulation of metabolism. Physiol Rev 86: 465-514.
Dudas I, Elmaouhoub A, Mansuroglu T, Batusic D, Tron K, Saile B, Papoutsi M, Pieler T, Wilting J, Ramadori G. 2006. Prospero-related homeobox 1 (Prox1) is a stable hepatocyte marker during liver development, injury and regeneration, and is absent from "oval cells". Histochem Cell Biol 126: 549-562.

Dufour CR, Wilson BJ, Huss JM, Kelly DP, Alaynick WA, Downes M, Evans RM, Blanchette M, Giguère V. 2007. Genone-wide orchestration of cardiac functions by orphan nucler receptors ERR $\alpha$ and $\gamma$. Cell Metab 5: 345-356.

Dupuis J, Langenberg C, Prokopenko I, Saxena R, Soranzo N, Jackson AU, Wheeler E, Glazer NL, Bouatia-Naji N, Gloyn AL, et al. 2010. New genetic loci implicated in fasting glucose homeostasis and their impact on type 2 diabetes risk. Nat Genet 42: 105-113.

Feige JN, Auwerx J. 2007. Transcriptional coregulators in the control of energy homeostasis. Trends Cell Biol 17: 292-301.

Giguère V. 2008. Transcriptional control of energy homeostasis by the estrogen-related receptors. Endocr Rev 29: 677-696.

Harvey NL, Srinivasan RS, Dillard ME, Johnson NC, Witte MH, Boyd K, Sleeman MW, Oliver G. 2005. Lymphatic vascular defects promoted by Prox1 haploinsufficiency cause adult-onset obesity. Nat Genet 37: 1072-1081.

Johnson NC, Dillard ME, Baluk P, McDonald DM, Harvey NL, Frase SL, Oliver G. 2008. Lymphatic endothelial cell identity is reversible and its maintenance requires Proxl activity. Genes \& Dev 22: 3282-3291.

Kamiya A, Kakinuma S, Onodera M, Miyajima A, Nakauchi H. 2008. Prospero-related homeobox 1 and liver receptor homolog 1 coordinately regulate long-term proliferation of murine fetal hepatoblasts. Hepatology 48: 252-264.

Lee S, Kang J, Yoo J, Ganesan SK, Cook SC, Aguilar B, Ramu S, Lee J, Hong YK. 2009. Prox1 physically and functionally interacts with COUP-TFII to specify lymphatic endothelial cell fate. Blood 113: 1856-1859.

Lin J, Handschin C, Spiegelman BM. 2005. Metabolic control through the PGC-1 family of transcription coactivators. Cell Metab 1: 361-370.

Mootha VK, Handschin C, Arlow D, Xie X, St Pierre J, Sihag S, Yang W, Altshuler D, Puigserver P, Patterson N, et al. 2004. ERR $\alpha$ and GABPA $\alpha / \beta$ specify PGC- $1 \alpha$-dependent oxidative phosphorylation gene expression that is altered in diabetic muscle. Proc Natl Acad Sci 101: 6570-6575.

Qin J, Gao DM, Jiang QF, Zhou Q, Kong YY, Wang Y, Xie YH. 2004. Prospero-related homeobox (Proxl) is a corepressor of human liver receptor homolog-1 and suppresses the transcription of the cholesterol 7- $\alpha$-hydroxylase gene. Mol Endocrinol 18: 2424-2439.

Schreiber SN, Emter R, Hock MB, Knutti D, Cardenas J, Podvinec M, Oakeley EJ, Kralli A. 2004. The estrogen-related receptor $\alpha$ (ERR $\alpha)$ functions in PPAR $\gamma$ coactivator $1 \alpha$ (PGC-1 $\alpha$ )-induced mitochondrial biogenesis. Proc Natl Acad Sci 101: 6472-6477.

Smith AD, Sumazin P, Xuan Z, Zhang MQ. 2006. DNA motifs in human and mouse proximal promoters predict tissue-specific expression. Proc Natl Acad Sci 103: 6275-6280.

Song KH, Li T, Chiang JY. 2006. A Prospero-related homeodomain protein is a novel co-regulator of hepatocyte nuclear factor $4 \alpha$ that regulates the cholesterol 7 $\alpha$-hydroxylase gene. J Biol Chem 281: 10081-10088.

Sonoda J, Laganière J, Mehl IR, Barish GD, Chong LW, Li X, Scheffler IE, Mock DC, Bataille AR, Robert F, et al. 2007. Nuclear receptor ERR $\alpha$ and coactivator PGC-1 $\beta$ are effectors of IFN- $\gamma$ induced host defense. Genes \& Dev 21: 1909-1920.

Sosa-Pineda B, Wigle JT, Oliver G. 2000. Hepatocyte migration during liver development requires Prox1. Nat Genet 25: 254-255.

Wigle JT, Oliver G. 1999. Proxl function is required for the development of the murine lymphatic system. Cell 98: 769-778.

Xie X, Lu J, Kulbokas EJ, Golub TR, Mootha V, Lindblad-Toh K, Lander ES, Kellis M. 2005. Systematic discovery of regulatory motifs in human promoters and 3' UTRs by comparison of several mammals. Nature 434: 338-345.

Yamazaki T, Yoshimatsu Y, Morishita Y, Miyazono K, Watabe T. 2009. COUP-TFII regulates the functions of Proxl in lymphatic endothelial cells through direct interaction. Genes Cells 14: 425-434. 


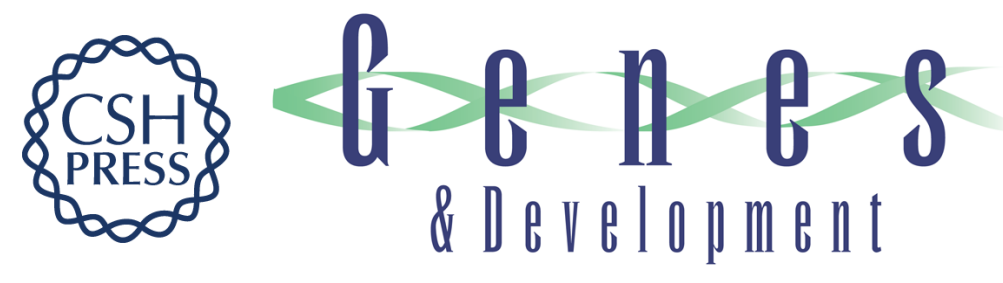

\section{The homeobox protein Prox1 is a negative modulator of ERR $\alpha / P G C-1 \alpha$ bioenergetic functions}

Alexis Charest-Marcotte, Catherine R. Dufour, Brian J. Wilson, et al.

Genes Dev. 2010, 24: originally published online March 1, 2010

Access the most recent version at doi:10.1101/gad.1871610

\section{Supplemental http://genesdev.cshlp.org/content/suppl/2010/02/24/gad.1871610.DC1 Material}

References This article cites 25 articles, 9 of which can be accessed free at: http://genesdev.cshlp.org/content/24/6/537.full.html\#ref-list-1

\section{License}

Email Alerting

Receive free email alerts when new articles cite this article - sign up in the box at the top Service 